\title{
Sound-induced artifact in cochlear blood flow measurements using the laser Doppler flowmeter
}

\author{
P.R. Thorne, A.L. Nuttall, F. Scheibe * and J.M. Miller \\ Kresge Hearing Research Institute, University of Michigan Medical Center, 1301 E. Ann Street, Ann Arbor, MI 48I09, U.S.A.
}

(Received 29 December 1986; accepted 25 September 1987)

\begin{abstract}
The laser Doppler flowmeter has been shown to give a response from the cochlea during high intensity acoustic stimulation which is not related to blood flow through the cochlea. The magnitude of this response depends upon the intensity and frequency of stimulation and the location of the probe on the cochlea. Evidence is presented that the response is derived from the vibration of cochlear tissue and/or the bony cochlear shell during acoustic stimulation.
\end{abstract}

Laser Doppler flowmeter: Blood flow: Cochlea; Sound stimulation

\section{Introduction}

The laser Doppler capillary perfusion monitor is a relatively new instrument for measuring blood flow. It provides a generally non-invasive technique of establishing qualitatively ${ }^{1}$ alterations in the perfusion of capillary beds and has now been used successfully in a variety of tissues and organs including skin (e.g. Schnizer et al., 1985), gastric mucosa (e.g. Kvietys et al., 1985) and the cochlea (e.g. Miller et al., 1983). The technique is well suited to measuring blood flow in the cochlea because of problems of measuring flow in such a small tissue using other methods. The cochlea is enclosed in bone which makes it difficult to gain access for study by other techniques such as hydrogen clearance (Maass et al., 1978) or

Correspondence to: P.R. Thorne (present address), Department of Pathology, University of Auckland School of Medicine, Private Bag. Auckland, New Zealand.

* Present address: ENT Clinic (Charité). Humboldt University, Berlin, GDR.

1 Attempts are being made to quantitatively relate the laser Doppler reading to blood flow in certain organs (e.g. Smits et al. 1986). This may also be possible for the cochlea of the guinea pig by comparison with indicator dilution or microsphere measurements of blood flow. plethysmography (Suga and Snow. 1969). The flow is small which leads to a need for large sample sizes when the microsphere method is used (Hultcrantz and Angelborg, 1978). Thus, it is not surprising that there is considerable interest in the use of the laser Doppler technique for measuring cochlear blood flow.

Recently, in a series of studies undertaken to investigate changes in blood flow through the cochlea during loud sound exposure (Thorne and Nuttall, 1987), we found that the sound stimulus induced a response of the laser Doppler that was also elicited in animals post-mortem. This 'artifactual' response tended to obscure any real dynamic changes in flow during the exposure of the animals to sound. Since there is an increasing use of the laser Doppler instrument for studying the inner ear circulation and sound is the most relcvant stimulus, it is important to understand this phenomenon.

In this paper we present a description of the characteristics of the sound-induced artifact in laser Doppler flowmeter measurements, its relationship to the sound stimulus and possible causes. The response was found to be highly tonotopic in that the maximum output of the flowmeter when the probe was placed on any particular turn of the 
cochlea, occurred when the ear was stimulated by a tone that maximally stimulates that region of the cochlea. The output increased with increasing stimulus intensity. The artifact also occurred in animals with low hematocrit so it does not appear to be related to red cell vibration. These findings imply that the flow 'artifact' response is due to movement of cochlear tissues during sound stimulation.

\section{Materials and Methods}

Experiments were performed on pigmented guinea pigs $250-400 \mathrm{~g}$ in weight. The animals were anesthetized with diazepam $(0.5 \mathrm{mg} / \mathrm{kg})$ and fentanyl $(0.32 \mathrm{mg} / \mathrm{kg})$, tracheostomized and placed in a head-holder. The cochlea was exposed by a ventral approach leaving the middle ear intact and the periosteum of the cochlea was removed with cotton pledgets.

All experiments reported here were performed with a Med-Pacific LD 5000 laser Doppler capillary perfusion monitor (Med-Pacific Corporation Inc., Seattle, WA, U.S.A.). The probe (1.75 mm diameter) was held in a micromanipulator and placed over the bone of the cochlear turn of interest (Fig. 1). The laser Doppler instrument was zeroed and the ' $D C$ ' values obtained (a measure of the tissue reflectance) were $1000 \pm 100 \mathrm{mV}$. The

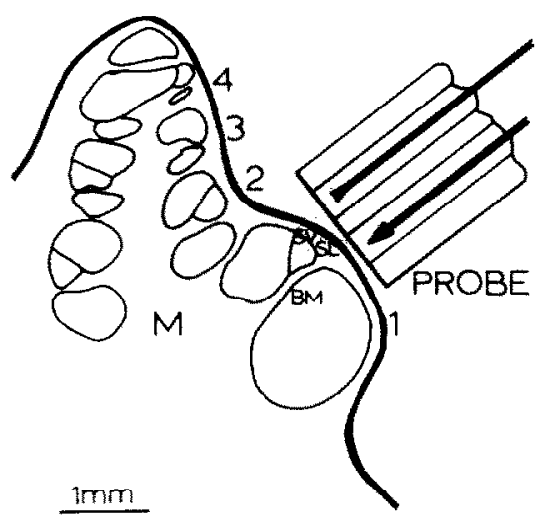

Fig. 1. A schematic diagram showing the four turns $(1,2,3,4)$ of the guinea pig cochlea. The location of the vascular beds in the spiral ligament (SL), stria vascularis (SV), basilar membrane (BM) and modiolus (M) are also shown. In this diagram the probe of the laser Doppler is placed over the lateral wall of the first turn. output of the laser Doppler flowmeter is proportional to the flux (i.e. the number of red cells in a given volume multiplied by their mean velocity) but because only relative changes can be measured all values for the output of the instrument in this paper are given in millivolts.

Animals were exposed to sound (either pure tones or white noise) through a $1 / 2$ inch condenser microphone (Brüel and Kjaer, Naerum, Denmark) inserted into a speculum sealed in the outer ear canal. Sound pressure levels were measured by the $1 \mathrm{~mm}(\mathrm{OD})$ probe-tube adapted to a $\frac{1}{2}$ inch condenser microphone (Brïel and Kjaer) inserted to within $1 \mathrm{~mm}$ of the tympanic membrane. The magnitude or other characteristics of the response of the laser Doppler instrument were measured relative to the sound pressure level at the ear drum at each tone frequency.

To test the effects of hematocrit on the laser Doppler response to loud sound, animals were hemodiluted (Hultcrantz and Nuttall, 1987) by withdrawing whole blood and infusing an equal volume of Dextran 75 (6\% solution in $0.9 \%$ saline). The hematocrit of blood drawn from a catheter inserted into the jugular vein was measured using a microcentrifuge. At the end of the experiment, the chest was opened, the vena cava was cut and the cardiovascular system was flushed with saline by intracardiac perfusion. Cochleae were removed from these animals, perfused with $2.5 \%$ glutaraldehyde in $0.1 \mathrm{M}$ phosphate buffer and then dissected in phosphate buffer. The entire organ of Corti, spiral ligament and stria vascularis were removed from the cochlea and mounted on glass slides and studied by Nomarski differential interference contrast microscopy. The density of red cells in the vessels of the lateral wall, basilar membrane and tympanic lip was qualitatively assessed using a scale of 0 (no red cells) to 5 (densely packed). Observations were made at 400 $\times$ magnification. The magnitude of the response during pure tone stimulation was measured at each hematocrit level and after the saline flush.

\section{Results}

The presentation of sound to the ear resulted in an increase in the flow output of the laser Doppler instrument regardless of whether the sound was a 


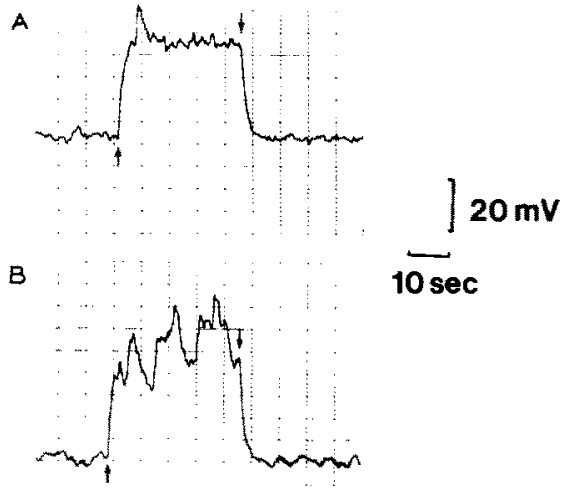

$13 \mathrm{kHz} ; 105 \mathrm{~dB}$ SPL

Fig. 2. The flow output of the Med-Pacific laser Doppler with the probe on the first turn during exposure (between arrows) of the live (A) and dead (B) animal to a pure tone of $13 \mathrm{kHz}$ at $105 \mathrm{~dB}$ SPL.

pure tone or wide-band noise. This response occurred abruptly when the sound was turned on and then returned to the baseline as rapidly when it was turned off (Fig. 2). A similar response could be elicited post-mortem, although, unlike the generally steady-state response in the live animal, the post-mortem response showed more variation in amplitude while the tone was on (Fig. 2). One animal was left for $3 \mathrm{~h}$ post-mortem during which time the magnitude of the artifact response to occasional testing by the same tone and intensity remained unchanged. The response was not observed if the probe was placed on the bone of the bulla and it was severely reduced when the ossicular chain was disarticulated.

The magnitude of the response at any particular location along the cochlea was related to both the frequency and the intensity of the sound stimulus. This was true for the living animal and post-mortem. If the probe was placed on the first cochlear turn, approximately $3-5 \mathrm{~mm}$ from the round window, and the animal exposed to tones at the same intensity a maximal response was achieved with a stimulus frequency of $12-16 \mathrm{kHz}$ (Fig. 3). In the second and third turns there was a maximal response at $4 \mathrm{kHz}$ and below $0.5 \mathrm{kHz}$, respectively (Fig. 3). At any frequency, the flowmeter voltage output increased in proportion to the sound pressure in decibels, once a threshold of approximately $80 \mathrm{~dB}$ SPL (Fig. 4) was exceeded.

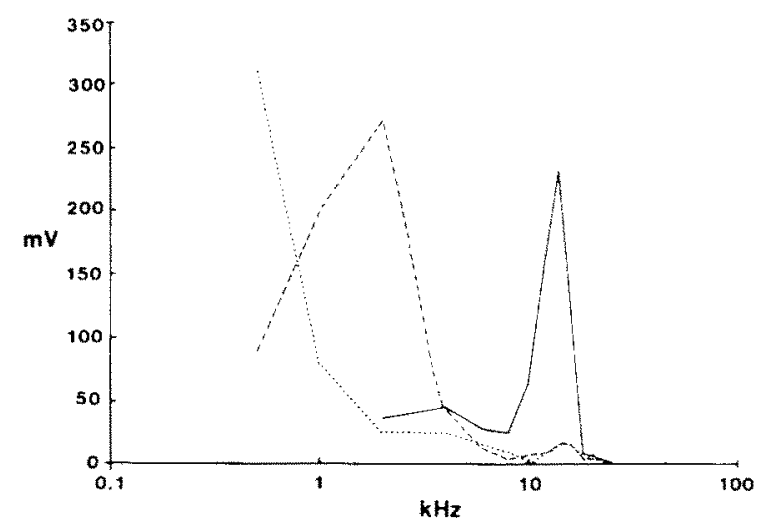

Fig. 3. Laser Doppler output $(\mathrm{mV})$ recorded from a cochlea post-mortem exposed to different frequencies $(\mathrm{kHz})$ at $110 \mathrm{~dB}$ SPL when the probe was placed on the 1 st (solid line). 2nd (dashed line) or 3rd (dotted line) turn.

Four hemodilution experiments were conducted in order to test whether the artifactual response was the result of red cell vibration during the sound stimulus or due to the movement of other structures within the cochlea. In each case the response to sound of the same frequency and intensity either increased in magnitude or remained unchanged as the hematocrit declined. There was no change in the response in two animals but it increased substantially in the other

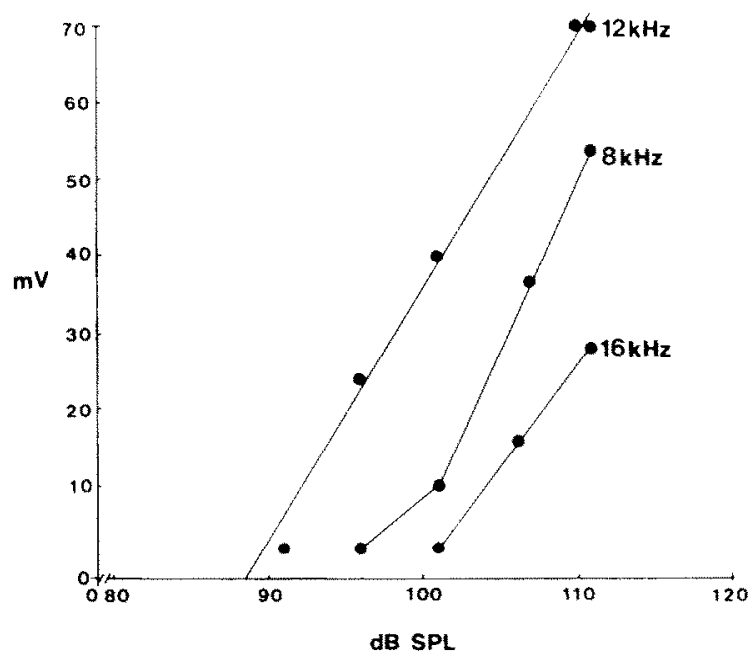

Fig. 4. The increase in laser Doppler output recorded from the 1st cochlear turn with increasing intensity of pure tone exposure. The peak response with the probe in this location occurred at a frequency of $12 \mathrm{kHz}$. 
two animals, relative to the values obtained before hemodilution, when they were perfused with saline to remove red cells. All the cochleae of these animals were studied histologically. Vessels of the basilar membrane the spiral ligament and stria vascularis of three animals were generally devoid of red cells (grade $0-1$ ). Occasional red cells were observed in the vessels throughout the cochlea but these were scattered throughout the vasculature and not concentrated at any particular location. The vessels throughout the cochlea of the remaining animal were packed with red cells (grade 4-5).

\section{Discussion}

The laser Doppler flowmeter is becoming a popular technique for studying cochlear blood flow since it is basically non-invasive, apart from the surgery needed to open the auditory bulla, and can be used to make many continuous measurements of experimental manipulations. This report, however, demonstrates that the laser Doppler flowmeter may give an erroneous flow response when used to study dynamic changes in cochlear blood flow during loud sound exposure.

The laser Doppler flowmeter works on the principle that the emitted light is scattered randomly by tissue structures and is shifted in frequency by moving objects such as red cells (Nilsson et al., 1980; Bonner and Nossal, 1981). Because the increase in the flow output of the laser Doppler instrument with loud sound exposure occurs generally in a similar fashion both post-mortem and in the live animal, it is obviously not a real indication of blood flow. The response is observed only when the probe is placed on the cochlea and not on the bone of the bulla. Additionally, during all experiments care was taken to ensure that there was no fluid or blood beneath the probe that could be vibrating during the sound exposure. It would thus appear that this artifactual response is due to the vibration of some structures within the cochlea.

Acoustic stimulation of the cochlea results in a travelling wave along the basilar membrane (Békésy, 1960). The cochlea is tonotopically organized such that the location of maximal displacement of the cochlear partition depends upon the frequency of the sound stimulus (Békésy, 1960;
Wilson and Johnstone, 1975). High frequencies stimulate the basal turn and the location of maximal stimulation moves apically as stimulus frequency decreases. The artifactual response of the laser Doppler flowmeter also showed a tonotopic relationship along the cochlea. The maximal response, on each cochlear turn, occurred at an approximate frequency that would be expected to Inaximally stimulate the region beneath the probe, according to the place-frequency map (Wilson and Johnstone, 1975). Isointensity curves in Fig. 3 indicate this tonotopic character but these curves should not be confused with neural or basilar membrane tuning curves.

There are several possible explanations for the source of the artifact.

(i) Red cells in the vessels of the basilar membrane or spiral ligament may be set into vibration during sound exposure. Vibration of red cells would induce a Doppler shift which would be measured and displayed as a change in flow by the laser Doppler instrument. The tonotopic relationship of the artifactual response could be consistent with this hypothesis. However, it would have been expected that the response would have disappeared or at least diminished substantially as the hematocrit was reduced and the red cells washed out of the cochlea with saline. This generally did not occur in the present study, assuming that the reduced red cell density, seen in cochlear vessels by histology reflects the situation in vivo. Other considerations also militate against a red cell vibration hypothesis. Further study (see (iii) below) of the nature of the change in back-scattercd light indicates that most of the artifact is due to amplitude modulation of the light and not Doppler shift. Doppler shifts which could be imparted by the velocities of structures vibrating at acoustic frequencies would be far above the electronic performance bandwidth of the instrument. Additionally, high viscous forces would be experienced by such vibrating red cells, thus limiting their movement.

(ii) The vibration of membranous structures within the cochlea, such as the basilar membrane, organ of Corti, or spiral ligament could be responsible for the artifactual response. This possibility is consistent also with the tonotopic character of the artifact. The expected spectral change 
should be at least partially a Doppler shift. This is because light would be scattered by the ncarby transparent, vibrating tissue elements and the direction of the light incident on tissues would be as randomized as it is for blood cells. While it is very difficult to rule out the vibration of internal structures by experiment, this possibility seems unlikely given the amplitude modulated character of the spectral change in back-scattered light and the preliminary results of some further studies (see (iii) below).

(iii) The response could be due to vibration of the bony cochlear shell. Preliminary experiments (Nuttall, Dolan and Thorne, 1987, Unpubl.) indicate that the source of the flow artifact is most likely amplitude modulation of light reflected from the vibrating surface of the cochlea. The spectrum of the photocurrent of the laser Doppler flowmeter was measured directly when the probe was on the cochlea and the ear exposed to pure tones. This revealed an amplitude modulated component of the light received by the instrument. Although it is not a Doppler shift, the component frequency (i.e. the acoustic frequency) falls within the analysis bandwidth of the instrument and is thus measured as an increase in flow. The amplitude modulated component of the photocurrent could be shown to exist on the surface of the cochlea when it is made opaque with a thin layer of solidified cement adhered to the cochlear bone. Under this condition it was found that the surface of the cochlea appears to have a topographical vibration pattern consistent in a general way with the location of expected maximum basilar membrane amplitude. If an opaque material did not adhere to the surface of the cochlea, or was soft and compliant so as not to transmit vibration, the artifactual response was greatly diminished. We interpret these results as favoring a hypothesis that it is the surface of the bony cochlea which generates the artifact. We are not aware of any other study which has demonstrated a topographical vibration of the cochlea which is consistent in a general pattern to the internal tonotopic vibration of the basilar membrane. This finding may be interpreted as a type of bone conduction due either to the inherent mechanical characteristics of the cochlea shell (in the guinea pig) or to the "localized' loss of energy from the cochlear fluids to the bone.
The presence of an artifact in the flow measurements during sound stimulation provides problems for the study of dynamic changes in blood flow in the cochlea during exposure to sounds in excess of $80-85 \mathrm{~dB}$ SPL. The Periflux (Perimed, Stockholm, Sweden) and TSI Laserflo (TSI Corporation, St. Paul, MN, U.S.A.) instruments have electronic filters which filter out certain frequencies in the optical laser Doppler signal. These filters may reduce or eliminate the artifact but only in response to sound at frequencies above about $4 \mathrm{kHz}$, depending on the specific design of the instrument. Thus, these instruments may be useful for studies of the lower cochlear turns. In a recent study of the effects of noise on cochlear blood flow (Thorne and Nuttall, 1987), the sound was turned off at particular intervals in order to make stable blood flow measurements without the artifact. Such a method assumes that changes in blood flow during the quiet period reflect those during the sound exposure. Currently the 'correct' technique for the use of laser Doppler flowmeters in these studies must depend on the goal of the study coupled with the knowledge of the function and design of the particular instrument.

In conclusion, this study demonstrates that the laser Doppler flowmeter can give a response during acoustic stimulation that does not reflect blood flow but instead may be due to vibration of cochlear structures. Thus, the use of the laser Doppler instrument to study cochlear blood flow during acoustic stimulation must be undertaken with caution and awareness of possible erroneous results.

\section{Acknowledgements}

The authors wish to thank J. Hardy and L. Logan for assistance in preparing this manuscript. We acknowledge the gift of Fentanyl from Janssen Pharmaceuticals Ltd. This research was supported by a USPHS International Research Fellowship no. TWO 3473 to P.R.T. and NIH grant 17731 to A.L.N.

\section{References}

Békésy, G. von (1960) Experiments in Hearing. McGraw-Hill. New York. 
Bonner, R. and Nossal, R. (1981) Model for laser Doppler measurements of blood flow in tissue. Appl. Opt. 20. 2097-2107.

Hultcrantz, E. and Angelborg, C. (1978) Cochlear blood circulation studied with microspheres. Oto-Rhino-I aryngology $40,65-76$.

Hultcrantz, E. and Nuttall, A.L. (1987) Effect of hemodilution on cuchlear blood flow measured by laser Doppler flowmetry. Am. J. Otolaryngol. 8, 16-22.

Kvietys, P.R., Shepherd, A.P. and Granger, D.N. (1985) Laser-Doppler, $\mathrm{H}_{2}$ clearance, and microsphere estimates of mucosal blood flow. Am. J. Physiol. 249, G221-G227.

Maass, B., Baumgartl, H. and Lubbers, D.W. (1978) Lokale $\mathrm{pO}_{2}$ - und $\mathrm{pH}_{2}$ Messungen mit Mikrokoaxialnadelektroden an der Basalwindung der Katzencochlea nach akuter oberer zervikaler Sympathektomie. Arch. Otorhinolaryngol. 221. 269-284.

Miller, J.M., Marks, N.J. and Goodwin, P.C. (1983) Laser Doppler measurements of cochlear blood flow. Hear. Res. $11,385-394$.
Nilsson, G.E., Tenland, T. and Oberg, P.A. (1980) Evaluation of a laser Doppler flowmeter for measurement of tissue blood flow. IEEE Trans. Biomed. Eng. 27, 597604.

Schnizer, W., Erdl, R., Schops, P. and Seichart. N. (1985) The effects of external $\mathrm{CO}_{2}$ application on human skin microcirculation investigated by laser Doppler flowmetry. Int. J, Microcirc. Clin. Exp. 4, 343-350.

Smits, G.J., Roman, R.J. and Lombard, J.II. (1986) An evaluation of laser Doppler flowmetry as a measure of tissue blood flow. J. Appl. Physiol. 61, 666-672.

Suga, F. and Snow, J.B. (1969) Cholinergic control of cochlear blood flow. Ann. Otol. Rhinol. Laryngol. 78, 1081-1090.

Thorne, P.R. and Nuttall, A.L. (1987) Laser Doppler measurements of cochlear blood flow during loud sound exposure in the guinea pig. Hear. Res. 27, 1-10.

Wilson, J.P. and Johnstone, J.R (1975) Basilar membrane and middle ear vibration in guinea pig measured by capacitive probe. J. Acoust. Soc. Am. 57, 705-723. 